\title{
BPH: Frühe Kombinationstherapie verringert Progressionsrisiko
}

- Unbehandelt oder falsch behandelt haben Patienten mit einer benignen Prostatahyperplasie (BPH) ein hohes Risiko für die Entwicklung von Komplikationen wie akuter Harnverhalt und BPH-bedingten Operationen. Ein besonders hohes Progressionsrisiko haben Männer mit einem Prostatavolumen $\geq 30 \mathrm{ml}$, PSA-Werten $\geq 1,5 \mathrm{ng} / \mathrm{ml}$ und einem Alter über 50 Jahre, so Prof. Mark Emberton, London. Inzwischen gebe es solide Studiendaten, die zeigen, dass diese Patienten schon bei moderater Symptomatik von einer Kombinationstherapie aus dem a-Blocker Tamsulosin und dem 5a-Reduktasehemmer Dutasterid (Duodart ${ }^{\circledR}$ ) profitieren können.

Der Stellenwert der Kombinationstherapie mit Tamsulosin/Dutasterid ist vor allem auf die Ergebnisse der CombAT (Combination of Avodart and Tamsulosin)-Studie zurück- zuführen. In die dreiarmige Studie wurden 4.844 Patienten mit moderaten bis schweren BPH-Symptomen (International Prostate Symptom Score, IPSS, $\geq 12$ ), einem Prostatavolumen $\geq 30 \mathrm{ml}$ und einem PSA-Wert $\geq 1,5 \mathrm{ng} / \mathrm{ml}$ eingeschlossen. Sie erhielten entweder Tamsulosin (0,5 mg/d), Dutasterid $(0,5 \mathrm{mg} / \mathrm{d})$ oder eine Kombination beider Substanzen. Die Kombination Tamsulosin/ Dutasterid führte im Vergleich zu den Monotherapien zu einer stärkeren Symptomreduktion. Mit steigendem Prostatavolumen nahm der Vorteil der Kombinationstherapie deutlich zu.

Besonders wichtig sei aber die Reduktion des Progressionsrisikos, betonte Emberton. Nach 48 Monaten betrug die Gesamtinzidenz für akuten Harnverhalt oder BPH-bedingte Operationen 4,2\% im Kombinationsarm gegenüber 5,2\% unter Dutasterid und
$11,9 \%$ unter Tamsulosin (jeweils $p<0,0001$ ). Damit sank relative Risiko für die BPH-bedingten Komplikationen um 19,6\% gegenüber einer Dutasterid-Monotherapie und um $65,8 \%$ gegenüber Tamsulosin. Das Risiko einer Progression (Verschlechterung des IPSS um $\geq 4$ Punkte) der BPH wurde durch die Kombinationstherapie um 41,3\% gegenüber Tamsulosin und um 35,2\% gegenüber Dutasterid reduziert (jeweils $p<0,001$ ). Die Kombination von Tamsulosin plus Dutasterid zeigte ein gutes Verträglichkeits- und Sicherheitsprofil.

Abdol A. Ameri

Satellitensymposium "Evidence and rationale for initial treatment of patients with symptomatic BPH at risk of progression“ im Rahmen des EAU-Kongresses,

Paris, 25. Februar 2012;

Veranstalter: GlaxoSmithKline, München

\section{Erfolgreiche Langzeittherapie der Gicht}

— Die Therapie der Gicht beruht auf zwei Säulen: der Entzündungshemmung und Analgesie im akuten Anfall sowie der langfristigen Senkung des Serumharnsäurespiegels. Im akuten Gichtanfall ist Colchicin das Mittel der Wahl, um die Entzündung einzudämmen. Aufgrund des erheblichen Nebenwirkungsrisikos, besonders der häufigen Brechdurchfälle bei höheren Dosen, sollte die Initialdosis nur 1,2 mg betragen, gefolgt von einer weiteren Dosis von 0,6 mg etwa eine Stunde später. Voraussetzung ist eine normale Nierenfunktion. Für die Analgesie und Entzündungshemmung können zudem nicht steroidale Antirheumatika (NSAR) wie Indometacin, Ibuprofen, Diclofenac und Etoricoxib eingesetzt werden. Bei bestehender Niereninsuffizienz sind Kortikoide eine gute Alternative. Nicht geeignet ist Acetylsalicylsäure (ASS), weil sie dosisabhängig die Ausscheidung von Harnsäure beeinflusst: In geringen Dosen verlangsamt ASS die Harnsäureausscheidung, erst ab einer Dosis von mehr als $3 \mathrm{~g} / \mathrm{d}$ dreht sich dieser Effekt um.

Langfristig steht die Senkung der Serumharnsäure im Vordergrund, um die Neubildung von Uratkristallen zu verhindern und weitere Gelenkerosionen aufzuhalten. Stan- dard hierfür ist bislang das Urikostatikum Allopurinol. Unter der Therapie wird die Zielsetzung, den Harnsäurespiegel auf unter $6 \mathrm{mg} / \mathrm{dl}$ beziehungsweise $360 \mu \mathrm{mol} / \mathrm{l} \mathrm{zu}$ senken, allerdings nur in etwa einem Fünftel der Fälle und bei hoher Dosierung erreicht. Deutlich effizienter ist offenbar der neue selektive Xanthinoxidasehemmer Febuxostat (Adenuric ${ }^{\oplus}$ ). Der Wirkstoff hat keine Purinstruktur und ist deshalb eine gute Behandlungsalternative. Zudem kann er auch bei leichter bis mittelschwerer Niereninsuffizienz ohne Dosisanpassung eingesetzt werden. Vorsicht ist allerdings bei schwerer Leberinsuffizienz geboten, da Febuxostat hepatisch abgebaut wird. Weil sich in Studien zudem eine erhöhte Rate kardiovaskulärer Ereignisse gezeigt hat, ist die Anwendung bei ischämischer Herzkrankheit und dekompensierter Herzinsuffizienz derzeit nicht empfohlen.

Kathrin Sommer

"GichtAkademie Fokus 2012 - Fortschritte in Diagnose und Therapie der Arthritis urica", Berlin, 9.-11. März 2012;

Veranstalter: Berlin-Chemie, Berlin
Kongress-App auch für Android-Geräte

- Farco-Pharma bietet ab sofort die beliebte "UroCongress"-App, die es bislang nur für iPhone-Nutzer gab, jetzt auch im Google Play Store (ehemals Android-Market) für Android Smartphones an. Die App „UroCongress" bietet dem Verwender Informationen zu nationalen und internationalen urologischen Kongressen, Tagungen und Symposien. Neben Basisinformationen zu den einzelnen Veranstaltungen wie Datum, Ort und Veranstalter besteht auch die Option, Veranstaltungen nach Themenschwerpunkten auszuwählen. Vorteilhaft ist die Möglichkeit, eine persönliche Favoritenliste erstellen zu können. Darüber hinaus ist ein Routenplaner integriert.

Die „UroCongress"-App wird kontinuierlich für das aktuelle Veranstaltungsjahr und die Folgejahre aktualisiert.

Nach Informationen von

Farco-Pharma, Köln 